\title{
Panorama des cordons optiques actifs ou AOC - active optical cables
}

\author{
D'éléments passifs, les cordons optiques rentrent désormais dans la famille des équipements actifs. Pourquoi et \\ comment cette évolution ? Quels sont les principaux domaines d'applications des AOC ? Quels sont les principaux \\ fabricants ? Panorama de ce marché émergeant et fort prometteur...
}

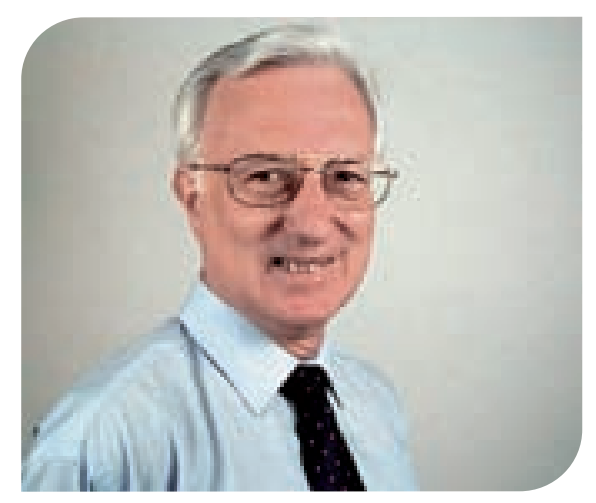

$>$ Jean-Michel MUR

Président du Club optique

Jm.mur@orange.fr

M arché récent, créé en 2007 par de jeunes pousses américaines sans usines (fabless start-up), le secteur «Active optical cabling " a balbutié de 2008 à 2010. Il est, désormais, en croissance : de 137 millions de dollars en 2011, il pourrait dépasser les 500 millions de dollars en 2013 pour atteindre 1,4 milliard de dollars en 2015 (source : IGI-Information Gatekeepers Inc., janvier 2011). Mais ces TPE ou PME n'ont pas les ressources pour suivre ce développement annoncé. Aussi, cela aiguise les appétits des gros et les grandes manœuvres ont commencé. Le leader français de la connectique, $\mathrm{FCl}$, a tiré le premier, en février 2010, en achetant la société allemande Merge Optics. En mai 2010, le leader mondial Tyco Electronics acquiert la gamme de produits optoélectroniques de Zarlink. En novembre 2010, Mellanox annonce l'achat de son concurrent Voltaire, finalisation prévue courant $1^{\mathrm{er}}$ trimestre 2011. Et, tout récemment, en janvier 2011, Molex a confirmé l'achat de la partie AOC de
Luxtera. Tout indique que cela devrait continuer en 2011. Mais qu'est-ce qu'un $\mathrm{AOC}$ et d'où vient cet engouement?

\section{Description d'un AOC}

Un cordon optique actif ou Active optical cable (AOC) est un lien de câblage entre équipements électroniques qui accepte les mêmes entrées et sorties électriques qu'un cordon en cuivre actif mais dont le support entre connecteurs est une fibre optique. Ainsi, les $\mathrm{AOC}$ sont composés de quatre parties : les émetteurs-récepteurs optiques (optical transceivers), les connecteurs assurant la liaison avec les équipements électroniques, les fiches optiques au bout des fibres optiques et les fibres elles-mêmes.

Les émetteurs-récepteurs assurent les conversions électrique vers optique et optique vers électrique à chaque extrémité tout en respectant la compatibilité avec les interfaces électriques standard. Pour $80 \%$ de l'offre, les émetteurs sont des lasers VCSEL travaillant dans la fenêtre des $850 \mathrm{~nm}$ et les récepteurs sont des photodiodes PIN. Pour $20 \%$ des AOC, les émetteurs sont des lasers émettant dans les fenêtres de 1310 ou $1550 \mathrm{~nm}$ et les récepteurs sont des photodiodes à avalanche.

Les connecteurs qui seront insérés dans les emplacements dédiés des serveurs, routeurs, commutateurs, etc. répondent à des normes précises. La plupart de ces «normes» sont concoctées par des industriels réunis sous l'acronyme MSA (multiple source agreement). Parmi ceux-ci figurent Agilent, Finisar, Fujikura, Hitachi, IBM, Molex, Tyco Electronics... Les produits les plus courants sont les SFP (small form factor pluggable) pour des débits

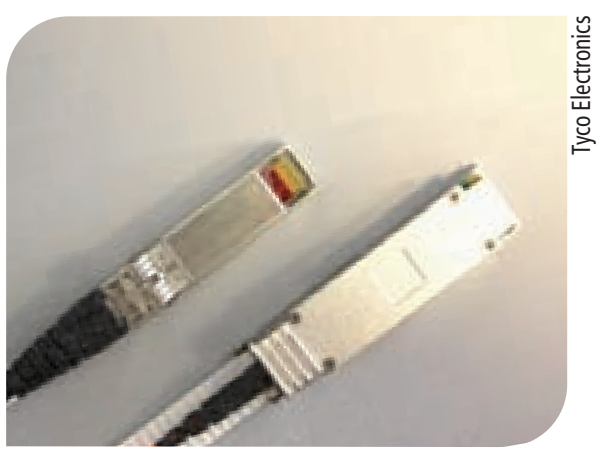

Figure 1. En mai 2010, le leader mondial, Tyco Electronics a acquis la gamme de produits optoélectroniques de Zarlink.

de 1 à 5 Gbit/s et leur évolution SFP+ pour les débits jusqu'à $10 \mathrm{Gbit} / \mathrm{s}$. Au-delà, on trouve le QSFP (quad SFP), nouvelle génération d'interfaces à 4 canaux de $10 \mathrm{Gbit} / \mathrm{s}$, soit $40 \mathrm{Gbit} / \mathrm{s}$ en cumulé. L'interface autorisant le plus fort débit est le CXP (C, en base hexadécimale veut dire $12, \mathrm{XP}=$ extended capability pluggable). C'est une interface qui offre 12 canaux à $10 \mathrm{Gbit} / \mathrm{s}$ par canal afin de fournir une bande passante totale de $120 \mathrm{Gbit} / \mathrm{s}$ théorique et de $96 \mathrm{Gbit} / \mathrm{s}$ en réel. À noter qu'un AOC peut avoir des connecteurs de types différents à chacune de ses extrémités. Par exemple, un CXP de 12 canaux à une extrémité peut être composé de 3 QSFP à 4 canaux chacun à l'autre extrémité. Les fiches optiques sont généralement de deux types : soit les fiches de modèle LC (Lucent connector) mondialement connues et utilisées en version simplex ou en version duplex, soit des fiches basées sur la connectique MPO (multifiber push-on), idéale pour les fibres optiques en ruban. Certains fabricants proposent une variante de MPO baptisée MTP (mechanical transfer push-on) présentant une plus faible perte d'insertion et donc moins de perte de puissance du signal 
associée à une meilleure tenue mécanique. Deux versions courantes pour ces fiches MPO ou MTP : une pour les fibres ruban de 12 fibres optiques, I'autre pour 24 fibres optiques.

Quant aux fibres optiques, c'est le produit débit $x$ distance visé qui définira le choix. Pour de faibles distances - de 1 mètre à 33 mètres - avec des débits de 1 à $10 \mathrm{Gbit} / \mathrm{s}$, I'AOC pourra être constitué de fibres optiques multimodales de diamètre de cœur de 50 ou $62,5 \mu \mathrm{m}$ et de bande passante de $200 \mathrm{MHz} . \mathrm{km}$. Au-delà de cette trentaine de mètres et pour des débits de $10 \mathrm{Gbit} / \mathrm{s}$, on emploie des fibres multimodales de $50 \mu \mathrm{m}$ de cœur mais avec des bandes passantes plus élevées, jusqu'à $3500 \mathrm{MHz} . \mathrm{km}$. Ces fibres multimodales sont aussi connues sous la dénomination OM1, OM2, OM3 et OM4 (norme ISO/IEC 11801). Plus l'indice est élevé, plus le produit débit $x$ distance est élevé. II est à noter que les fibres installées dans certains $A O C$ permettent d'aller plus loin que la distance normalisée, jusqu'à 550 mètres comme avec la fibre MaxCap550 de Draka ou la Pretium550 de Corning. Avec le développement de la connectique MPO/ MTP, les câbliers proposent des câbles de 12 fibres optiques soit sous forme de câble rond soit sous forme de câble ruban.

Lorsque la distance s'allonge, pouvant atteindre plusieurs kilomètres, les $A O C$ sont construits sur des fibres optiques unimodales (single mode fibre - SMF), de $9 \mu \mathrm{m}$ de cœur, et dont la bande passante offre des produits de débit $x$ distance bien supérieurs. Pour les installations type cam- pus, zones portuaires, aéroportuaires... soit pour des distances jusqu'à quatre kilomètres, la tendance vient à utiliser les fibres normalisées OS1 ou les récentes fibres de type G. 657 pour leurs très faibles rayons de courbure. Pour les très longues distances, c'est la classique fibre G.652 qui conserve sa prééminence.

\section{D'où vient cet engouement pour les AOC?}

Jusqu'alors, les connexions entre calculateurs, serveurs, routeurs, commutateurs, etc. étaient classiquement en câbles de cuivre qui de passifs sont devenus actifs en incorporant émetteurs et récepteurs de signaux de transmission. Mais trois facteurs modifient la donne: I'augmentation de la puissance de calcul des ordinateurs, la montée en très haut débit de transmission et l'accroissement des distances entre équipements. Cela oblige les intégrateurs et les installateurs à mettre en place des liens en fibre optique, eu égard aux qualités intrinsèques de ce support : produit débit $x$ distance sans commune mesure entre câbles en cuivre et ceux en fibre optique, insensibilité aux interférences électromagnétiques, taille plus faible, poids plus léger, etc. Voyons l'évolution des trois facteurs...

L'augmentation de la puissance de calcul des centres de calcul hautement performant type HPC (high performance computing) est telle que l'on ne parle plus que de calculateurs pétaflopiques,
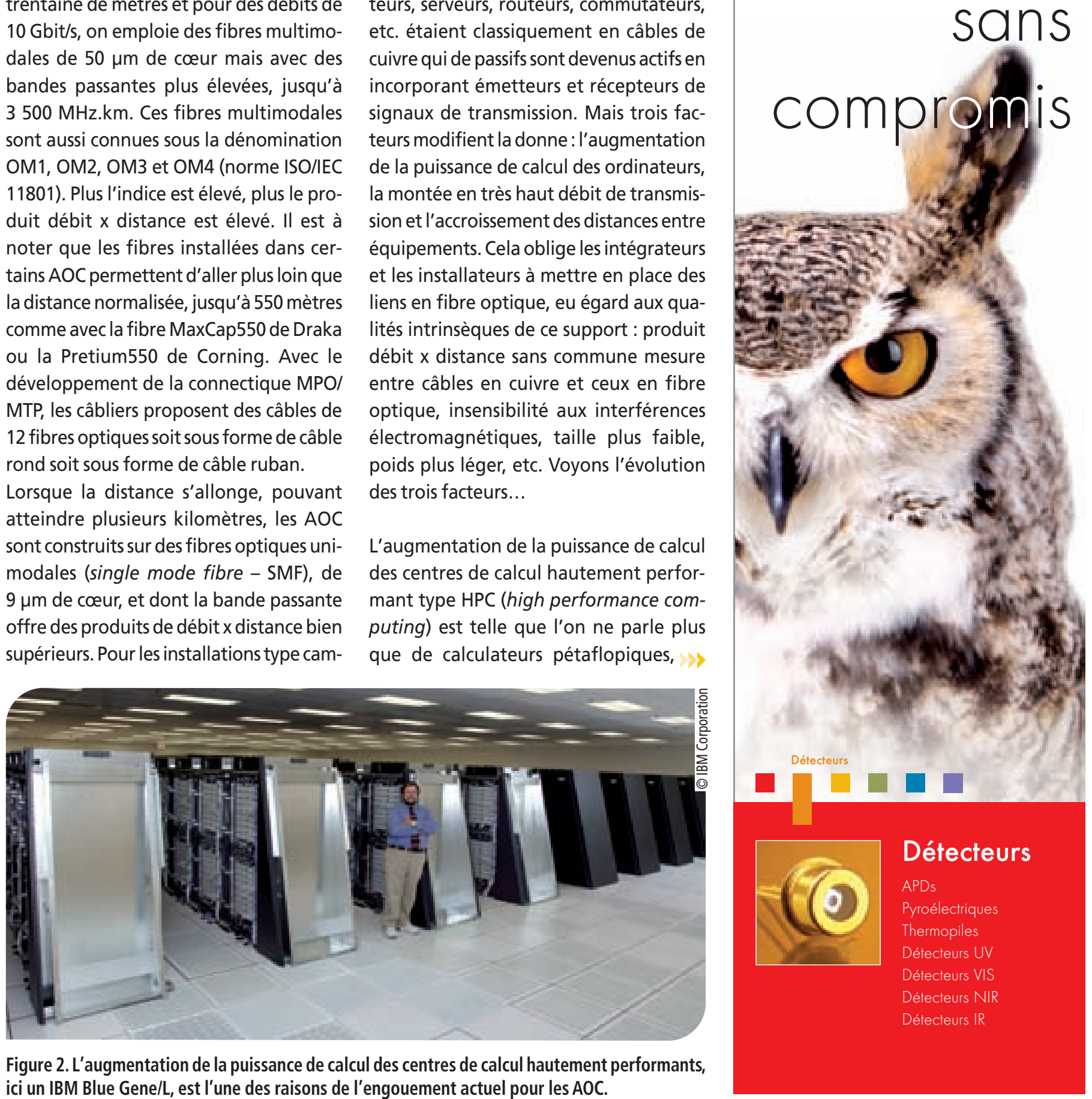

Figure 2. L'augmentation de la puissance de calcul des centres de calcul hautement performants, ici un IBM Blue Gene/L, est l'une des raisons de l'engouement actuel pour les AOC. 
c'est-à-dire dont la puissance de calcul est supérieure au pétaflop (un million de milliards d'opérations en virgule flottante par seconde). Deux exemples : le champion du monde, Tianhe-1A chinois, et le champion européen, Tera 100 de Bull. Le Tianhe-1A est une machine installée à Tianjin et conçue par I'Université Nationale des Technologies de Défense dont la puissance brute atteint les 4,7 pétaflops. Elle utilise une architecture mixant 112 armoires dédiées aux serveurs de calcul comprenant un total de 14336 processeurs classiques et de 7168 cartes NVIDIA Tesla M2050. Le tout est assisté par 262 To de mémoire vive et 2 Po d'espace disque. Le Tera 100, quant à lui, nouveau supercalculateur du CEA-DAM (Direction des applications militaires du Commissariat à l'énergie atomique), conçu en coopération avec Bull, affiche une puissance de 1,05 pétaflop. Cette machine est aisément exploitable, ce qui en fait une solution très généraliste. Elle se présente sous la forme d'une grappe de serveurs (computer cluster) composée de 4370 serveurs Bullx série $S$, soit un total de 17480 processeurs Intel Xeon, épaulés par 300 To de mémoire vive et une capacité de stockage de 20 Po. Tianhe-1A, Tera 100 et autres... avec l'explosion du nombre de ports à connecter, voilà un domaine rêvé pour les $A O C$.

La montée en très haut débit, vers les réseaux gigabitaires, est vraie pour tous les protocoles. Les exemples les plus connus sont les protocoles communément installés dans les centres informatiques (data center) que sont InfiniBand, Fiber Channel et Ethernet (voir encadré $n^{\circ} 1$ ). Tout ceci est tiré par le déploiement en multi-sites d'applications de stockage de données - gestion mondiale de fichiers avec le stockage virtuel entre sites de grands volumes " petascale global storage architecture » ; d'applications de rattrapage de désastre - services financiers, marchés de change à temps de réponse rapide ; de bases de données et entrepôts de données (data warehouse) en miroir et multi-sites avec partage de l'information et accès en temps réel ; d'applications médicales réparties dont le déploiement d'imagerie à haute résolution, etc. Un protocole tel que PCle (peripheral component interconnect express) n'est pas en reste et 2011 voit se développer la génération 3 qui atteint $8 \mathrm{Gbit} / \mathrm{s}$ par lien soit $64 \mathrm{Gbit} / \mathrm{s}$ cumulé pour les 8 liens parallèles. À niveau moindre, la montée des débits se retrouve aussi dans le grand public à travers les réseaux domestiques (HAN home area network) et les systèmes électroniques domestiques dont l'architecture (HES - home electronic systems) est définie dans la norme ISO/CEI 14543-522:2010. L'interconnexion peut être assurée par des cordons optiques universels de type LightPeak d'Intel (produits annoncés en septembre 2009 et attendus, enfin, pour mi-2011) ou par des cordons HDMI pour les nouveaux téléviseurs (HD, $3 \mathrm{D}, 4 \mathrm{~K} . .$.$) et leur évolution vers le home$ cinema. Là aussi, les débits sont tirés vers le haut avec l'annonce en février 2011, par Time Interconnect Ltd, de son HDMI Hyper Speed transmettant $21 \mathrm{Gbit} / \mathrm{s}$.

Enfin, il faut noter l'accroissement des distances entre équipements tiré par deux facteurs qui sont l'augmentation de la taille des centres informatiques et leur sécurisation. Pour la taille, la dernière

\section{Parlez-vous InfiniBand ?}

Les AOC pour InfiniBand se trouvent essentiellement dans l'application rattachant directement les unités de disques aux serveurs (direct attached storage DAS) avec un mode de connexion SCSI (small computer system interface) ou SATA (serial advanced technology attachment). Mais qu'il semble lointain le temps du premier InfiniBand SDR (simple data rate) au débit de 2,5 Git/s. La profession est passée allégrement au DDR (double data rate) à 5 Gbits/s, en 2005, puis au QDR (quad data rate) à $10 \mathrm{Gbit} / \mathrm{s}$, en 2008 . Désormais, en 2011, sont proposés des produits pour le FDR (fourteen data rate) à $14 \mathrm{Gbit} / \mathrm{s}$ et pour

I'EDR (enhanced data rate) à $26 \mathrm{Gbit} / \mathrm{s}$. Ce dernier permet ainsi d'atteindre $312 \mathrm{Gbit} / \mathrm{s}$ sur 12 fibres optiques parallèles !

Attention! En langage InfiniBand, $20 \mathrm{Gbit} / \mathrm{s}$ veut dire $20 \mathrm{Gbit} / \mathrm{s}$ en cumulé. En effet, avec la transmission en parallèle sur plusieurs fibres optiques, $20 \mathrm{Gbit} / \mathrm{s}$ peut vouloir dire $8 \times 2,5 \mathrm{Gbit} / \mathrm{s}$ ou 4 x $5 \mathrm{Gbit} / \mathrm{s}$ ou $2 \times$ $10 \mathrm{Gbit} / \mathrm{s}$ ! De fait, deux données doivent être prises en compte :

1. De quel InfiniBand s'agit-il ? SDR, DDR, QDR, FDR ou EDR ?

2. De combien de fibres optiques parallèles les cordons optiques actifs se composent-ils ? 4 fibres, 8 fibres ou 12 fibres ?

InfiniBand Débit total en fonction du nombre de fibres optiques parallèles

\begin{tabular}{|c|c|c|c|c|c|}
\hline Type & Débit par lien & $\mathbf{4 f ø}$ & $\mathbf{8} ø$ & $12 \mathrm{fø}$ \\
\hline SDR & $2,5 \mathrm{Gbit} / \mathrm{s}$ & $10 \mathrm{Gbit} / \mathrm{s}$ & $20 \mathrm{Gbit} / \mathrm{s}$ & $30 \mathrm{Gbit} / \mathrm{s}$ \\
\hline DDR & $5 \mathrm{Gbit} / \mathrm{s}$ & $20 \mathrm{Gbit} / \mathrm{s}$ & $40 \mathrm{Gbit} / \mathrm{s}$ & $60 \mathrm{Gbit} / \mathrm{s}$ \\
\hline QDR & $10 \mathrm{Gbit} / \mathrm{s}$ & $40 \mathrm{Gbit} / \mathrm{s}$ & $80 \mathrm{Gbit} / \mathrm{s}$ & $120 \mathrm{Gbit} / \mathrm{s}$ \\
\hline FDR & $14 \mathrm{Gbit} / \mathrm{s}$ & $56 \mathrm{Gbit} / \mathrm{s}$ & $112 \mathrm{Gbit} / \mathrm{s}$ & $168 \mathrm{Gbit} / \mathrm{s}$ \\
\hline EDR & $26 \mathrm{Gbit} / \mathrm{s}$ & $104 \mathrm{Gbit} / \mathrm{s}$ & $208 \mathrm{Gbit} / \mathrm{s}$ & $312 \mathrm{Gbit} / \mathrm{s}$ \\
\hline
\end{tabular}




\section{HORIBA}

Scientific

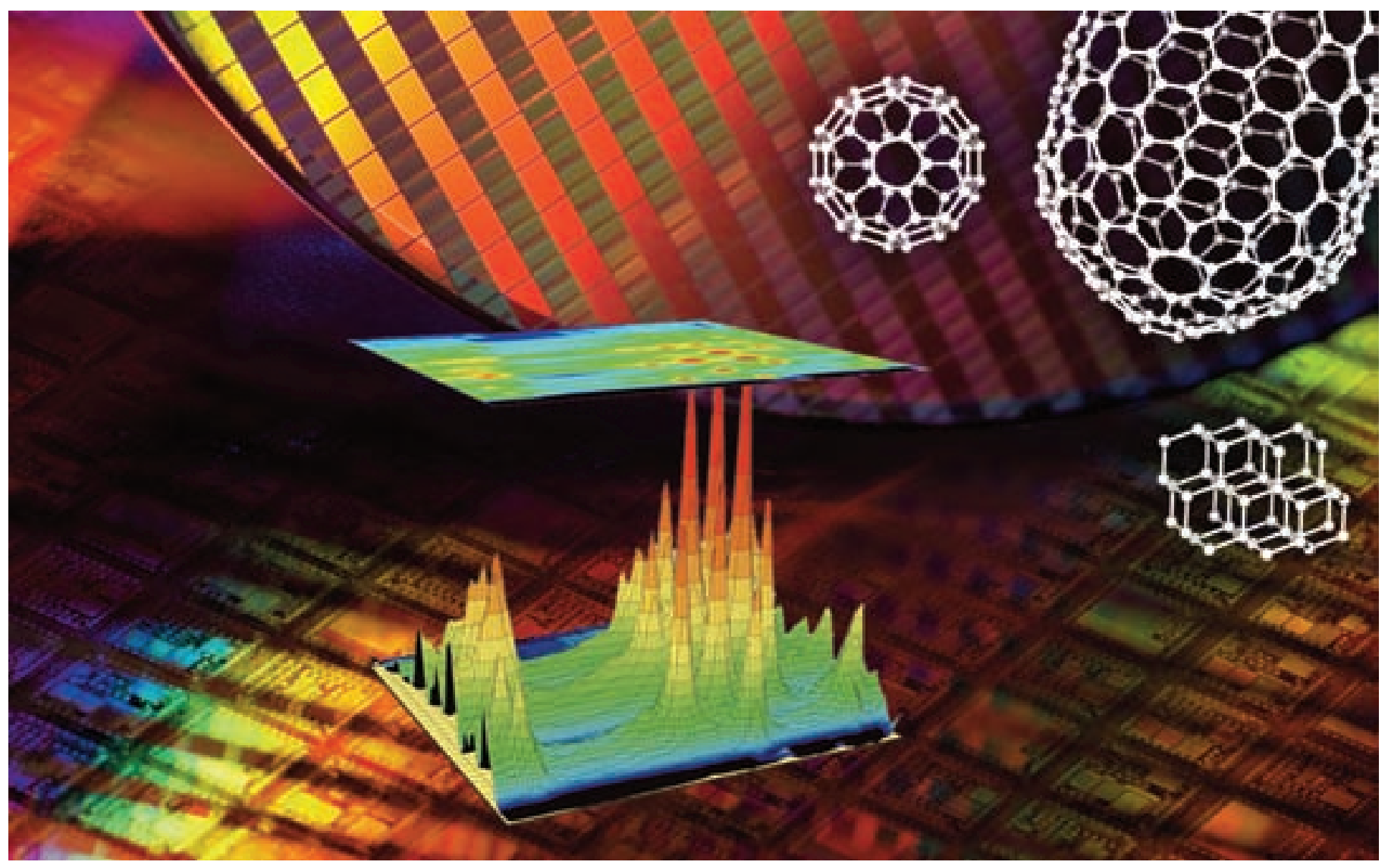

\section{Nano-réalité !}

Relevez le défi de la caractérisation des nano-structures, de la détermination des informations chimiques structurales et dimensionnelles à l'échelle du nanomètre.

Spectroscopie Raman - Spectrofluorimétrie

Ellipsométrie Spectroscopique

Analyse de taille de particules

Contactez-nous au +33(0)164541300.

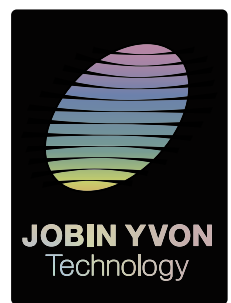


Parmi les caractéristiques discriminantes on note le type de connectique, les débits supportés, les distances couvertes... toutes choses facilement identifiables ou mesurables.

D'autres caractéristiques sont plus difficilement vérifiables mais annoncées fièrement par les fournisseurs. Par exemple, ils insistent tous sur le taux d'erreur de transmission (bit error rate - BER) qui de $10^{-12}$ en cuivre atteint de meilleures performances, de l'ordre de $10^{-15}$, en fibre optique. D'autres chiffrent les temps de latence dus aux conversions électriqueoptique ou inversement. Généralement, ces temps sont de l'ordre de 2 nanosecondes. Cependant, Hitachi Cable annonce 300 picosecondes par extrémité pour son $\mathrm{AOC}$ et Emcore descend à 275 picosecondes. Il est à noter que cette donnée présente de l'intérêt pour des distances très courtes mais peut être considérée comme superflue dès que le cordon dépasse une vingtaine de mètres. Certaines autres caractéristiques demandent une lecture attentive des notices. C'est le cas, par exemple, lorsqu'il faut prendre en compte des obligations légales : les $A O C$ installés dans un bâtiment recevant du public devront avoir un câble de fibres optiques classé LSZH (low smoke zero halogen), et ce n'est pas le cas pour tous. De même, certains annoncent fièrement un 6/6 quant au respect de la norme RoHS et sans plomb, d'autres ne semblent pas aussi formels.

Parmi les sujets qui peuvent fâcher, on trouve essentiellement des facteurs physiques pour la connectique tels que l'extrême précision demandée aux dimensions, la résistance aux efforts de traction, la bonne tenue même après plusieurs cycles de branchement-débranchement, etc. C'est pourquoi certains grands industriels de l'informatique prennent cela en argumentation en précisant que leurs solutions ne sont garanties que si, et seulement si, leurs propres AOC sont installés. Il va sans dire que cela a un coût beaucoup plus important pour le client. Autre astuce ou stratégie marketing (!), modifier un tant soit peu la norme sous prétexte d'amélioration. Ainsi un grand fabricant de commutateurs a créé, à côté des
SFP, un SFP miniaturisé, le mSFP, ainsi qu'un mini SFP LC, le tout aux dimensions légèrement réduites. II présente cette compacité comme avantage majeur assurant la connexion de ses châssis avec des AOC dédiés.

\section{Un mot sur les acteurs}

Développement du marché oblige, les acteurs se bougent. Parmi les principaux intervenants, les jeunes pousses élargissent leurs champs ou se font racheter. Exemples d'élargissement de gammes : la californienne Alpen IO attaque le marché européen via la société slovaque Sylex ; une autre californienne, Cosemi, spécialiste des photodiodes, propose un AOC CXP 120 Gbit/s et un HDMI ; Emcore, ex Intel ICC, utilise son savoir-faire en émetteurs-récepteurs pour proposer un AOC pour InfiniBand et $10 \mathrm{GbE}$; la taïwanaise Genista complète sa gamme d'une douzaine d'émetteurs-récepteurs par des $A O C$ de plusieurs types dont un de 120 kilomètres; GigOptix, spécialiste d'émetteurs-récepteurs optiques pour les télécoms commercialise un AOC pour le $40 \mathrm{GbE}$ et un pour le $100 \mathrm{GbE}$; Mellanox, israélo-californiennne, complète ses cartes

\section{Parallèle entre réseaux de stockage et Fiber Channel}

Les AOC pour Fiber Channel se retrouvent principalement dans l'application réseau de stockage (storage area network-SAN). Les deux types de Fiber Channel ont également vu leurs débits respectifs exploser. La génération «Base 2 » a vu la normalisation du doublement régulier de son débit passant de 2 à 4 puis 8 et, aujourd'hui, à $16 \mathrm{Gbit} / \mathrm{s}$. Scénario identique pour la géné-

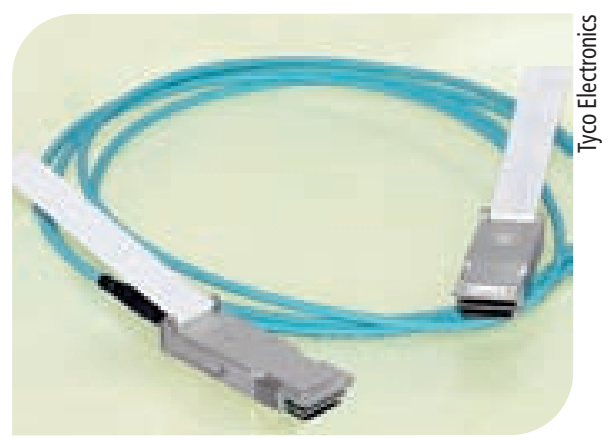

Figure 3. Le modèle Paralight-40Gb de Tyco Electronics.

pour InfiniBand et Ethernet par un AOC QSFP répondant à la norme IEEE 802.3 10Gbase-SR (short reach) pour les entreprises et IEEE 802.3 10Gbase-LR (long reach) pour les exploitants de réseaux.

Pour les grandes entreprises, on peut noter Avago Technologies avec $3 \mathrm{AOC}$ pour I'InfiniBand à $20 \mathrm{Gbit} / \mathrm{s}$ et $40 \mathrm{Gbit} / \mathrm{s}$ en cumulé sur 4 liens parallèles; la division High Speed Connections de $\mathrm{FCl}$ avec les produits Merge Optics dont un AOC CXPCXP à 24 fibres optiques pour transmettre $120 \mathrm{Gbit} / \mathrm{s}$; Finisar et ses trois gammes $\mathrm{d}^{\prime} \mathrm{AOC}$ - Laserwire à $10 \mathrm{Gbit} / \mathrm{s}$, Quadwire à $40 \mathrm{Gbit} / \mathrm{s}$ et $\mathrm{C}$. wire à $150 \mathrm{Gbit} / \mathrm{s}$; Hitachi Cable et sa gamme d'AOC InfiniGreen

\begin{tabular}{|c|c|c|c|c|c|}
\hline $\begin{array}{c}\text { Génération } \\
\text { des SAN }\end{array}$ & Année & $\begin{array}{c}\text { Nombre de } \\
\text { ports d'un SAN }\end{array}$ & $\begin{array}{c}\text { Surface } \\
\text { informatique }\end{array}$ & $\begin{array}{c}\text { Débits des liens } \\
\text { Fiber Channel (FC) }\end{array}$ & $\begin{array}{c}\text { Câblage } \\
\text { structuré }\end{array}$ \\
\hline Première & 1998 & 10 & $100 \mathrm{~m}^{2}$ & $1 \mathrm{Gbit} / \mathrm{s}$ & Non \\
\hline Deuxième & 2002 & 100 & $1000 \mathrm{~m}^{2}$ & $2 \mathrm{Gbit} / \mathrm{s}$ & Non \\
\hline Troisième & 2005 & 1000 & $10000 \mathrm{~m}^{2}$ & 4 ou $10 \mathrm{Gbit} / \mathrm{s}$ & Parfois \\
\hline Quatrième & 2008 & 10000 & $10000 \mathrm{~m}^{2}$ & 8 ou $10 \mathrm{Gbit} / \mathrm{s}$ & Souvent \\
\hline Cinquième & 2011 & 100000 & $>10000 \mathrm{~m}^{2}$ & $\begin{array}{c}\text { 16 ou } 20 \mathrm{Gbit} / \mathrm{s} \mathrm{en} \mathrm{FC} \\
10 \mathrm{Gbit} / \mathrm{s} \mathrm{en} \mathrm{FCoE} \text { Obligatoire }\end{array}$ \\
\hline
\end{tabular}

(1) La surface est souvent limitée par les besoins en énergie et climatisation. Le nombre de ports au $\mathrm{m}^{2}$ se densifie compte tenu de la miniaturisation et de l'intégration des composants électroniques. ration « Base 10 » passant de 10 à 20 puis à $40 \mathrm{Gbit} / \mathrm{s}$.

Les commutateurs Fiber Channel sont spécifiques. Aussi, pour utiliser des commutateurs classiques, le marché propose d'encapsuler Fiber Channel dans Ethernet (Fiber Channel over Ethernet - FCoE). Ainsi, cela permet la mixité réseau SAN et réseau local d'entreprise (local area network - LAN). 


\section{Des normes..}

Les fibres optiques multimodales existent avec des diamètres de cœur de $50 \mu \mathrm{m}$ ou $62,5 \mu \mathrm{m}$ et des bandes passantes allant de $200 \mathrm{MHz} . \mathrm{km}$ à près de $3500 \mathrm{MHz}$.km. Pour s'y retrouver, des normes existent ou, plutôt, coexistent en fonction des

organismes de normalisation qui les ont éditées.

Exemples de correspondance de caractéristiques quasi similaires malgré des noms différents dans la galaxie des documents normatifs.

Normes ou organismes Type de fibre optique multimodale (optical fiber multimode)

\begin{tabular}{|c|c|c|c|}
\hline ISO/CEI 11801 & OM 4 & OM 3 & OM 2 \\
\hline CEI 60793-2-10 & A1a.3 & A1a.2 & A1a.1 \\
\hline TIA/EIA & 492AAAD & 492AAAC \& B & 492AAAB \& A \\
\hline UIT & G.651 & G.651 & G.651 \\
\hline
\end{tabular}

ISO = Organisation internationale de normalisation

$\mathrm{CEI}=$ Commission électrotechnique internationale ou IEC (international electrotechnical commission)

TIA = telecommunications industry association - EIA = electronic industries alliance

UIT = Union internationale des télécoms ou ITU (international telecommunications union)

dont un cordon CXP à 12 fibres pour $120 \mathrm{Gbit} / \mathrm{s}$ en cumulé ; Intel et ses essais de lancement - depuis septembre 2009 du cordon optique universel LightPeak ; Molex avec l'offre AOC de Luxtera, entre autres pour fonds de paniers et liens carte à carte, et son tout nouveau zQSFP+ annoncé en février 2011 ; Siemon a une offre, Moray, à 4 liens à $10 \mathrm{Gbit} / \mathrm{s}$ par lien pour les classiques InfiniBand, Fiber Channel et Ethernet mais, surtout, cet $A O C$ supporte la quatrième version du protocole Myrinet, appelée Myri-10G, protocole de réseau à $10 \mathrm{Gbit} / \mathrm{s}$ assurant I'interconnexion multiserveurs et interopérable, du point de vue physique, avec Ethernet; Tyco Electronics est présent sur ce marché grâce à l'achat de l'offre AOC de Zarlink qui est désormais commercialisée sous le nom ParaLight.

Et demain ? Les AOC pour Ethernet sont très présents dans les centres informatiques avec l'application stockage attachée au réseau (network attached storage NAS) composée de baies de disques dotées d'interfaces LAN afin d'être partagées par tous les serveurs ou autres équipements. Le développement des AOC pour Ethernet va se faire parallèlement à la croissance des débits proposés par les nouvelles normes. Le 17 juin 2010, I'arrivée de la norme IEEE 802.3 ba a permis le déploiement du $40 \mathrm{GbE}$, transmission en parallèle avec 4 canaux à $10 \mathrm{Gbit} / \mathrm{s}$
(40GBase-SR4) et du 100 GbE (100GBaseSR10), transmission en parallèle avec 10 canaux à $10 \mathrm{Gbit} / \mathrm{s}$. C'est d'autant plus intéressant que le groupe de travail de I'IEEE dédié à cette norme a travaillé en étroite collaboration avec le groupe de travail 15 de I'UIT pour s'assurer que ces nouveaux débits de l'Ethernet seront transportables sur les réseaux de transports optiques (optical transport network - OTN). Cette année 2011 devrait voir la ratification de la norme IEEE $802.3 \mathrm{bg}$ autorisant le $40 \mathrm{Gbit} / \mathrm{s}$ en transmission série sur une fibre optique unimodale. Pour les technophiles (geek), signalons que pour profiter à plein du passage à $10 \mathrm{Gbit} / \mathrm{s}$ et au-dessus, pour Ethernet et Fiber Channel, il est souhaitable d'abandonner le codage 8b/10b au profit du codage 64b/66b. Et pour les écologistes, notons que plus le débit augmente, plus le besoin d'énergie au bit transporté diminue.

Et après-demain ? Quelle évolution majeure pour le marché des AOC ? II semblerait qu'à moyen terme, en 2014, et d'après une étude d'Information Gatekeepers Inc., le marché en nombre d'unités d'AOC devrait être tiré par les applications personnelles. Il se répartirait entre les microordinateurs et produits périphériques (55\%), la télévision et produits connexes (33\%), l'informatique lourde (9\%) et les autres équipements électroniques (3\%). Rendez-vous dans 3 ans...

\section{OTRIOPTICS}

UN UNIVERS DE PRÉCISION

- Solutions complètes pour le test des systèmes optiques

- Interféromètres, goniomètres, collimateurs, autocollimateurs, bancs de tests, bancs de FTM.

- Mesure de focale, centrage, front d'onde, FTM, topographie.

- Applications en R \& D

et production.

- Développements spéciaux.

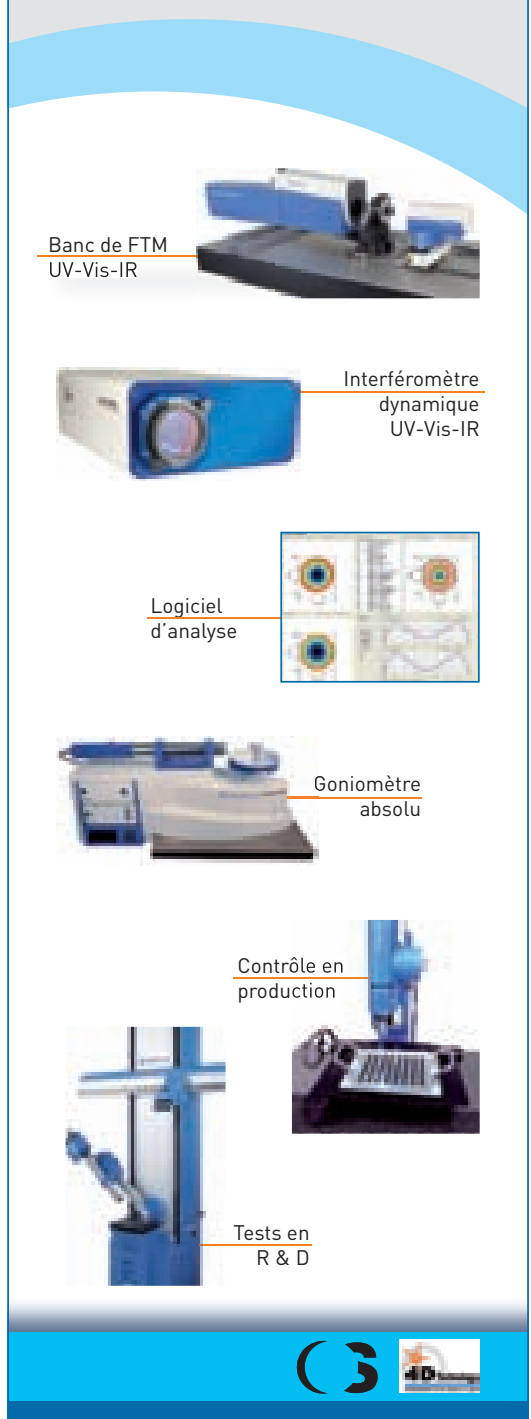

TRIOPTICS France

Tél: 0472440203

Fax: 0472440506

www.trioptics.fr 